\title{
A MATRIX METHOD FOR ELASTIC WAVE PROBLEMS
}

\author{
By L. KNopofF
}

\begin{abstract}
The solution to problems of elastic wave propagation in multilayered media, in which each layer is homogeneous and where the ensemble of layers has physical properties that vary only with one coordinate, may be given as the quotient of products of matrices. In the case of $S H$ waves, the matrices are of order two; in the case of $P-S V$ waves the matrices are of order four. The individual matrix elements are themselves determinants of order two or four in the two cases. The solution is obtained by means of Laplace's development by minors.
\end{abstract}

The solution to problems of wave propagation in inhomogeneous media in which the inhomogeneity varies with only one coordinate is much simplified if we consider the problems of wave propagation in a medium consisting of a large number of homogeneous layers. The homogeneous layers are taken to approximate the inhomogeneous medium as closely as desired. The eigenfunctions in homogeneous media can be written much more simply than those for inhomogeneous media. In compensation for the gain in simplicity of the elementary solutions, one must now solve a large number of simultaneous equations with a correspondingly large number of unknowns. This problem, too, has been overcome with the application of large computational equipment, at least for specific models of the inhomogeneities.

The solution to the problems of wave propagation in such inhomogeneous media can be expressed as the solution to a number of simultaneous equations, each expressing the values of the appropriate elementary solutions at the boundaries between adjacent layers. The solution to the simultaneous equations can be written in terms of a number of determinants constructed from the equations; it merely remains to evaluate the appropriate determinants.

When the number of layers becomes large, the order of the determinantal equations for the solution for plane harmonic waves becomes large. In the case of waves in a medium consisting of $N$ layers overlying a half-space and underlying a void, the determinants are of order $2 N+1$ for scalar (SH) elastic waves and $4 N+2$ for vector ( $P$ and $S V$ ) elastic waves. These determinants become cumbersome even for moderate values of $N$. Hence some method of decomposition of the determinants is appropriate. Since the determinants are mostly riagonal, the decomposition is not difficult. The evaluation of the determinaints can then be represented as a product of matrices. The advantages of using matrix representations in lengthy computer operations is evident since subroutines for matrix manipulation are in most computer libraries.

One procedure for the matrix manipulation of elastic wave problems has been devised by Thomson (1950) and corrected and elaborated by Haskell (1953). In this paper, an alternative matrix procedure for determinantal decomposition and solution is presented which is appropriate to machine computation.

We consider first the case of scalar ( $\mathrm{SH}$ ) waves in a medium consisting of $N$ flat homogeneous layers overlying a half-space and underlying a void. We order the interfaces $z_{0}, z_{1}, \cdots z_{N}$. Let the solution fic the displacement in the $n$th layer be 


$$
u_{n}=(-1)^{n} a_{n} \exp i\left(k x+\eta_{n} z-\omega t\right)+(-1)^{n} b_{n} \exp i\left(k x-\eta b_{n} z-\omega t\right)
$$

where $k^{2}+\eta_{n}{ }^{2}=\omega^{2} / \beta_{n}{ }^{2} . \beta_{n}$ is the shear wave velocity in the $n$th layer. If we apply the conditions for continuity of displacement and shear stress at the boundary $z_{n}$, we obtain the equations

$$
\begin{aligned}
& a_{n} \exp \left(i \eta_{n} z_{n}\right)+b_{n} \exp \left(-i \eta_{n} z_{n}\right)+a_{n+1} \exp \left(i \eta_{n+1} z_{n}\right) \\
& \quad+b_{n+1} \exp \left(-i \eta_{n+1} z_{n}\right)=0 \\
& \eta_{n} \mu_{n} a_{n} \exp \left(i \eta_{n} z_{n}\right)-\mu_{n} \eta_{n} b_{n} \exp \left(-i \eta_{n} z_{n}\right) \\
& +\mu_{n+1} \eta_{n+1} a_{n+1} \exp \left(i \eta_{n+1} z_{n}\right)-\eta_{n+1} \mu_{n+1} b_{n+1} \exp \left(-i \eta_{n+1} z_{n}\right)=0
\end{aligned}
$$

where $\mu_{n}$ is the shear modulus in the $n$th layer. Let the source of motion be located in the half-space, and let the incident waves be

$$
u_{N+1}^{(i)}=c(-1)^{N} \exp i\left(k x-\eta_{N+1} z-\omega t\right)
$$

where the medium occupies the region $z_{0}<z<\infty$. The equation expressing the boundary condition at $z_{N}$ is

$$
\begin{gathered}
a_{N} \exp i \eta_{N} z_{N}+b_{N} \exp \left(-i \eta_{N} z_{N}\right)+a_{N+1} \exp i \eta_{N+1} z_{N}=c \exp \left(-i \eta_{N+1} z\right) \\
\mu_{N} \eta_{N} a_{N} \exp i \eta_{N} z_{N}-\mu_{N} \eta_{N} b_{N} \exp \left(-i \eta_{N} z_{N}\right)+\mu_{N+1} \eta_{N+1} a_{N+1} \exp \left(i \eta_{N+1} z_{N}\right) \\
=-\mu_{N+1} \eta_{N+1} c \exp \left(-i \eta_{N+1} z_{N}\right)
\end{gathered}
$$

The solution for any of the coefficients $a_{n}, b_{n}$ can be expressed as a quotient of two determinants of order $2 N+1$. The denominator determinant is

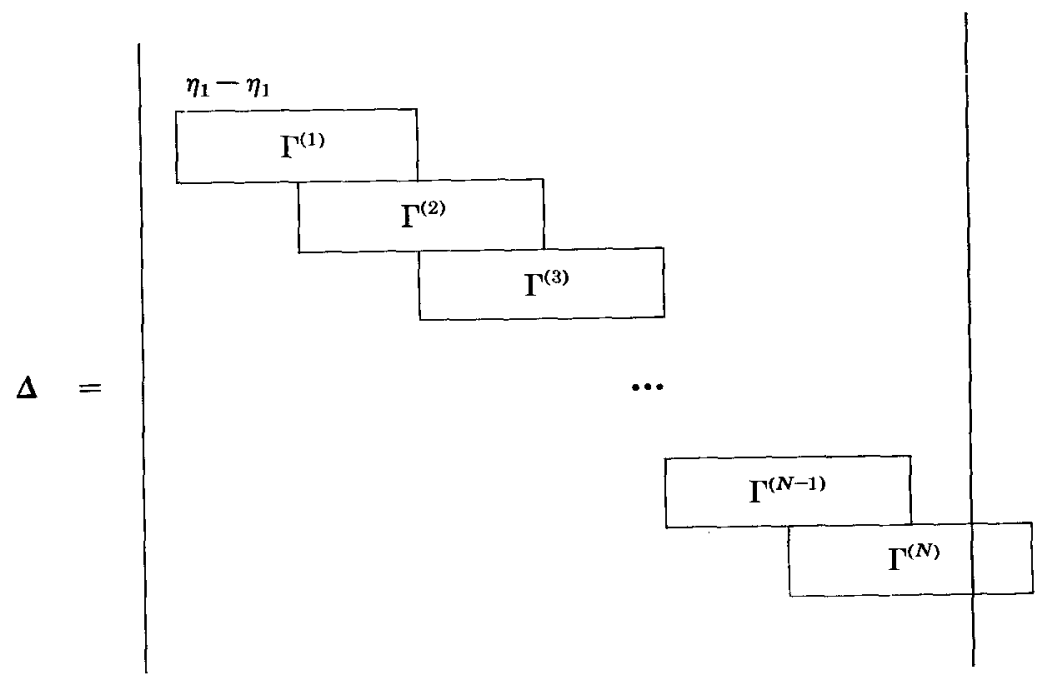


where the $2 \times 4$ submatrix $\Gamma^{(n)}$ is

$$
\begin{aligned}
& \Gamma^{(n)}=\left(\begin{array}{cccc}
\exp i \eta_{n} d_{n} & \exp -i \eta_{n} d_{n} & 1 & 1 \\
\mu_{n} \eta_{n} \exp i \eta_{n} d_{n} & -\mu_{n} \eta_{n} \exp \left(-i \eta_{n} d_{n}\right) & \mu_{n+1} \eta_{n+1} & -\mu_{n+1} \eta_{n+1}
\end{array}\right) \\
& d_{n}=z_{n}-z_{n-1}
\end{aligned}
$$

all the other elements not expressly specified are zero. The first three columns of $\Gamma^{(N)}$ are included in $\Delta$. The numerator determinant for the coefficient $a_{n}$ has the column $2 n-1$ replaced by the column

$$
\left(\begin{array}{c}
0 \\
0 \\
\cdots \\
0 \\
1 \\
-\eta_{N+1} \mu_{N+1}
\end{array}\right) c \exp -i\left(\eta_{N+1} z_{N}-\eta_{n} z_{n}\right)
$$

For coefficients $b_{n}$ the above column matrix is substituted in the $2 n$th column; the sign of the exponent of the second exponential multiplier in the column matrix is altered.

\section{Love Waves}

If we set $\Delta=0$, we obtain the eigenvalue equation for surface waves. In this section we decompose the determinant $\Delta$. Consider the typical matrix section $\Gamma^{(n)}$ composed of the non-zero elements of two rows of the larger matrix. We construct the matrix $T^{(n)}$ from $\Gamma^{(n)}$. The individual matrix element $T_{i j}^{(n)}$ is itself a determinant of order two, so constructed that it uses as the first column of the determinant the $i$ th column of the first two columns of $\Gamma^{(n)}$ and as its second column, the $j$ th column of the last two columns of $\Gamma^{(n)}, i=1,2 ; j=1,2$. Thus

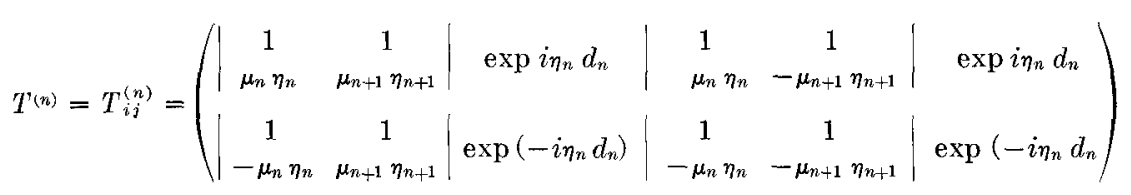

We further define the related matrix

$$
\bar{T}^{(n)}=T_{i j}^{(n)}=\left(\begin{array}{rr}
-T_{22}^{(n)} & T_{21}^{(n)} \\
T_{12}^{(n)} & -T_{11}^{(n)}
\end{array}\right)
$$

The barred notation implies that if $i=1, \bar{\imath}=2$, etc. The negative signs are introduced here to take account of negative cofactors that arise later. Thus $\Delta$ is, by Laplace's development by minors (Dickson, 1914),

$$
\Delta=T_{x_{1}}^{(0)} T_{x_{1} x_{2}}^{(1)} T_{x_{2} x_{3}}^{(2)} T_{\bar{x}_{3} x_{4}}^{(3)} \cdots T_{x_{N-1} x_{N}}^{(N-1)} T_{x_{N} 2}^{(N)} \exp \left(i \eta_{N+1} z_{N}\right)
$$

where $T_{1}^{(0)}=\eta_{1}, T_{2}^{(0)}=-\eta_{1}$ and where the repeated subscripts imply summation 
over the values 1,$2 ; x_{n}=1,2$. The last multiplier in eq. (4) is either $T_{\bar{x}_{N 2}}^{(n)}$ or $T_{x_{N} 1}^{(n)}$ according as $N$ is even or odd. The zeros of eq. (4) are found by assuming trial values of $k(\omega)$, substituting for $\eta_{n}$ from (1) into (4) and obtaining trial values of $\Delta$. The values of $k(\omega)$ corresponding to zero crossings of $\Delta$ are obtained by standard techniques. We may rewrite (4), dropping the subscripts to obtain

$$
\Delta=T^{(0)} \bar{T}^{(1)} T^{(2)} \bar{T}^{(3)} \cdots T^{(N-1)} \bar{T}^{(N)}
$$

The solution is thus seen to be a product of matrices.

If $k$ is given, then the angle of incidence of the incident wave in region $N+1$ is known, and the denominator determinant (5) has been evaluated as a first step in the computation of the coefficients $a_{n}, b_{n}$.

The procedure is a useful computation scheme, since matrix multiplication of second-order matrices is quite elementary; the matrix elements are given in eq. (3).

\section{The Displacement Coefficients-SH Waves}

If we replace the $2 n$th column by the inhomogeneous column, the determinant is no longer almost diagonal. However, by the usual Laplacian development, we may write

$$
\begin{aligned}
b_{n} \Delta & =-\left(T_{w_{1}}^{(0)} T_{\bar{w}_{1} \bar{w}_{2}}^{(1)} T_{w_{2} w_{3}}^{(2)} \cdots T_{\bar{w}_{n} 2}^{(n-1)}\right) R^{(n)} R^{(n+1)} \cdots R^{(N)} c \exp i\left(\eta_{n} z_{n}-2 \eta_{N+1} z_{N}\right) \\
& =-\left(T^{(0)} \bar{T}^{(1)} T^{(2)} \cdots \bar{T}^{(n-1)}\right) R^{(n)} R^{(n+1)} \cdots R^{(N)} c \exp i\left(\eta_{n} z_{n}-2 \eta_{N+1} z_{N}\right)
\end{aligned}
$$

where as before, the last element in the summed part of the numerator $T_{\bar{w}_{n} \overline{2}}^{(n-1)}$ may be $T_{w_{11}}^{(n-1)}$ according as $n$ is odd or even. The last element in $\Delta$, the denominator, may be $T_{\bar{x}_{N 2}}^{(N)}$ or $T_{x_{N} 1}^{(N)}$, as $N$ is even or odd. The multiplicative factors $R^{(p)}$ are the determinants of order two obtained from the last two columns of $\Gamma^{(p)}$ :

$$
R^{(p)}=\left|\begin{array}{cc}
1 & 1 \\
\mu_{p+1} \eta_{p+1} & -\mu_{p+1} \eta_{p+1}
\end{array}\right|=-2 \eta_{p+1} .
$$

In a similar way, if we replace the $(2 n-1)$ th column by the inhomogeneous column, the usual Laplacian development methods lead to

$$
\begin{aligned}
a_{n} \Delta & =\left(T_{w_{1}}^{(0)} T_{\bar{w}_{1} \bar{w}_{2}}^{(1)} T_{w_{2} w_{3}}^{(2)} \cdots T_{\bar{w}_{n-1} \overline{1}}^{(n-1)}\right) R^{(n)} R^{(n+1)} \cdots R^{(N)} c \exp i\left(\eta_{n} z_{n}-2 \eta_{N+1} z_{N}\right) \\
& =\left(T^{(0)} \bar{T}^{(1)} T^{(2)} \cdots \bar{T}^{(n-1)}\right) R^{(n)} R^{(n+1)} \cdots R^{(N)} c \exp i\left(\eta_{n} z_{n}-2 \eta_{N+1} z_{N}\right)
\end{aligned}
$$

with the usual admonitions regarding terminating the matrix products. In the cases for the solutions for $a_{1}, b_{1}$, the parenthesized expressions are simply $T_{2}^{(0)}$ and $T_{1}^{(0)}$ respectively.

\section{VECTOR WAVES}

Let the solutions for the displacement in the $n$th layer be

$$
\begin{aligned}
& u_{x}^{(n)}=\frac{\partial \phi^{(n)}}{\partial x}-\frac{\partial \psi^{(n)}}{\partial z} \\
& u_{z}^{(n)}=\frac{\partial \phi^{(n)}}{\partial z}-\frac{\partial \psi^{(n)}}{\partial z} .
\end{aligned}
$$


where

$$
\begin{aligned}
& (-1)^{n} \phi^{(n)}=a_{n} \exp i\left(k x+\zeta_{n} z-\omega t\right)+c_{n} \exp i\left(k x-\zeta_{n} z-\omega t\right) \\
& (-1)^{n} \psi^{(n)}=b_{n} \exp i\left(k x+\eta_{n} z-\omega t\right)+d_{n} \exp i\left(k x-\eta_{n} z-\omega t\right)
\end{aligned}
$$

and where $k^{2}+\zeta_{n}^{2}=\omega p_{n} /\left(\lambda_{n}+2 \mu_{n}\right)$ and $\eta_{n}$ is defined in (1).

If we apply the usual boundary conditions for continuity of normal stress and displacement across boundaries then the analogue to equation (2) is

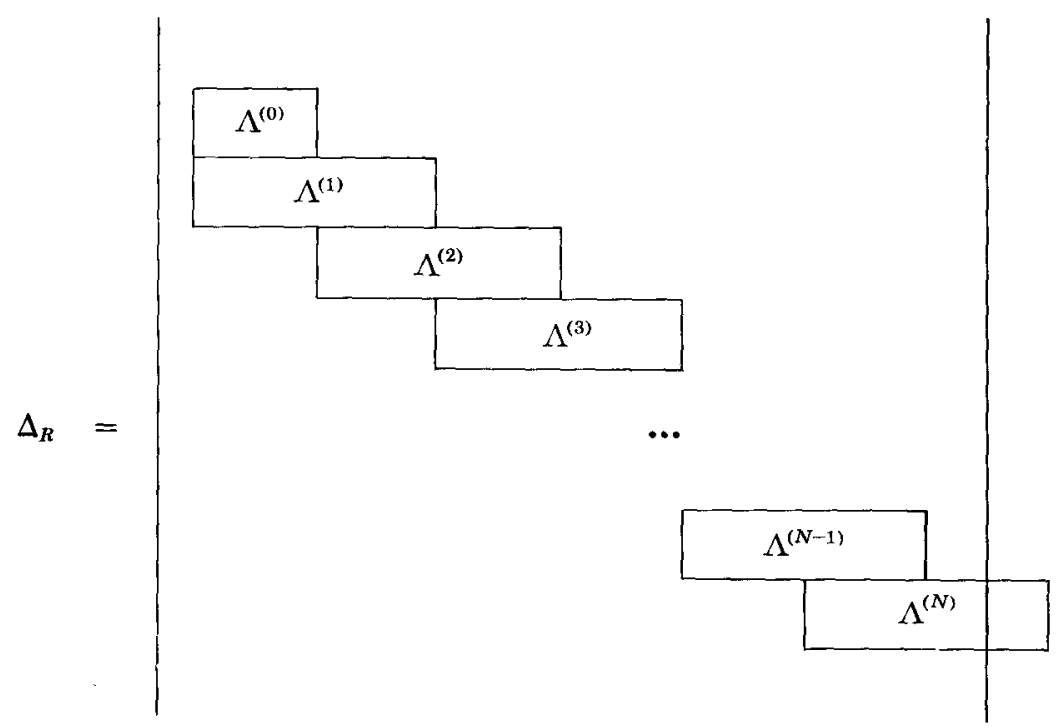

where the non-zero elements only are pictured; the $4 \times 8$ matrix $\Lambda^{(n)}$ is

$$
\begin{aligned}
& \Lambda^{(n)}=\left\{\begin{array}{ccc}
k e^{i \zeta_{n} d_{n}} & -\eta_{n} e^{-i \eta_{n} d_{n}} & k e^{-i \zeta_{n} d_{n}} \\
\zeta n e^{i \zeta_{n} d_{n}} & k e^{i \eta_{n} d_{n}} & -\zeta_{n} e^{-i \zeta_{n} d_{n}} \\
{\left[\left(\lambda_{n}+2 \mu_{n}\right) \zeta_{n}{ }^{2}+\lambda_{n} k^{2}\right] e^{i \zeta_{n} d_{n}}} & 2 \mu_{n} k \eta_{n} e^{i \eta_{n} d_{n}} & {\left[\left(\lambda_{n}+2 \mu_{n}\right) \zeta_{n}^{2}+\lambda_{n} k^{2}\right] e^{-i \zeta_{n} d_{n}}} \\
2 \mu_{n} k \zeta_{n} e^{i \zeta_{n} d_{n}} & \mu_{n}\left(k^{2}-\zeta_{n}^{2}\right) e^{i \eta_{n} d_{n}} & -2 \mu_{n} k \zeta_{n} e^{-i \zeta_{n} d_{n}}
\end{array}\right. \\
& \eta_{n} e^{-i \eta_{n} d_{n}} \quad k \quad-\eta_{n+1} \\
& k e^{-i \eta_{n} d_{n}} \quad \zeta_{n+1} \quad k \\
& -2 \mu_{n} k \eta_{n} e^{-i \eta_{n} d_{n}} \quad\left[\left(\lambda_{n+1}+2 \mu_{n+1}\right) \zeta_{n+1}^{2}+\lambda_{n+1} k^{2}\right] \quad 2 \mu_{n+1} k \eta_{n+1} \\
& \mu_{n}\left(k_{n}{ }^{2}-\zeta_{n}{ }^{2}\right) e^{-i \eta_{n} d_{n}} \quad 2 \mu_{n+1} k \zeta_{n+1} \quad \mu_{n+1}\left(k^{2}-\xi_{n+1}^{2}\right) \\
& \left.\begin{array}{cc}
k & \eta_{n+1} \\
-\zeta_{n+1} & k \\
{\left[\left(\lambda_{n+1}+2 \mu_{n+1}\right) \zeta_{n+1}^{2}+\lambda_{n+1} k^{2}\right]} & -2 \mu_{n+1} k \eta_{n+1} \\
-2 \mu_{n+1} k \zeta_{n+1} & \mu_{n+1}\left(k^{2}-\zeta_{n+1}^{2}\right)
\end{array}\right)
\end{aligned}
$$

and where $\Lambda^{(0)}$ is the $2 \times 4$ matrix formed of the last four columns and the two lowest rows of (8) with $n=0$ and where $\Lambda^{(N)}$ is given to the extent of the first six columns of (8). 


\section{RAYLEIGH WAVES}

We construct the matrix $F^{(n)}$ from $\Lambda^{(n)}$. The individual matrix element $F_{i j k l}^{(n)}$ is itself a determinant of order four, so constructed that it uses, as the first two columns of the determinants, two of the first four columns of $\Lambda^{(n)}$ and as the last two columns of the determinants two of the last four columns of $\Lambda^{(n)}$. There are six possible values of $i j: 12,13,14,23,24,34$. The same possible values of $k l$ exist. Thus the permissible values of $i j$ form a triangular matrix.

$$
F_{i j k l}^{(n)}=\left(\begin{array}{cccc}
0 & F_{12 k l}^{(n)} & F_{13 k l}^{(n)} & F_{14 k l}^{(n)} \\
0 & 0 & F_{23 k l}^{(n)} & F_{24 k l}^{(n)} \\
0 & 0 & 0 & F_{34 k l}^{(n)} \\
0 & 0 & 0 & 0
\end{array}\right)
$$

and similar triangular matrices can be written for other combinations of $k l$. In addition we define the related matrix $F_{\bar{i} j k l}^{(n)}$ derived from $F_{i j k l}^{(n)}$. Values of $i j$ corresponding to those given above are represented in the triangular matrix

$$
F_{\bar{i} j k l}^{(n)}=\left(\begin{array}{cccc}
0 & F_{34 k l}^{(n)} & -F_{24 k l}^{(n)} & F_{23 k l}^{(n)} \\
0 & 0 & F_{14 k l}^{(n)} & -F_{13 k l}^{(n)} \\
0 & 0 & 0 & F_{12 k l}^{(n)} \\
0 & 0 & 0 & 0
\end{array}\right)
$$

and a similar operation defines $F_{i j \bar{k} \tau}^{(n)}$. We may have a term such as $F_{i j k \bar{l}}^{(n)}$ arising from a doubled interchange of the type specified above; for example

$$
F_{1 \overline{3} 3 \overline{3}}^{(n)}=-F_{2412}^{(n)}=-\left|\begin{array}{cccc}
-\eta_{n} & \eta_{n} & k & -\eta_{n+1} \\
k & k & \zeta_{n+1} & k \\
2 \mu_{n} k \eta_{n} & -2 \mu_{n} k \eta_{n} & \left(\lambda_{n+1}+2 \mu_{n+1}\right) \zeta_{n+1}^{2}+\lambda_{n+1} k^{2} & 2 \mu_{n+1} k \eta_{n+1} \\
\mu_{n}\left(k^{2}-\zeta_{n}^{2}\right) & \mu_{n}\left(k^{2}-\zeta_{n}^{2}\right) & 2 \mu_{n+1} k \zeta_{n+1} & \mu_{n+1}\left(k^{2}-\zeta_{n+1}^{2}\right)
\end{array}\right|
$$

Then, if $\Delta_{R}$ is to vanish, a condition for surface waves, we write

$$
\begin{aligned}
\Delta_{R} & =T_{y_{1} y_{2}}^{(0)} F_{\tilde{y}_{1} \bar{y}_{2} \bar{y}_{3} \bar{y}_{4}}^{(1)} F_{y_{3} y_{4} y_{5} y_{6}}^{(2)} F_{\bar{y}_{5} \bar{y}_{6} \bar{y}_{7} \bar{y}_{8}}^{\left(\tilde{y}_{8}\right.} \cdots F_{\bar{y}_{2 N-3} \tilde{y}_{2 N-2} \bar{y}_{2 N-1} \bar{y}_{2 N}}^{(N-1)} F_{y_{2} N-1 y_{2} 1_{12}}^{(N)}=0 \\
& =T^{(0)} \bar{F}^{(1)} F^{(2)} \bar{F}^{(3)} \cdots \bar{F}^{(N-1)} F^{(N)}=0
\end{aligned}
$$

Depending upon evenness or oddness of $N$, the last two subscripts in $F^{(N)}$ may be either 12 or 34 . The matrix $T_{i j}^{(0)}$ is derived from $\Lambda^{0}$ in the same way as $T^{(n)}$ is derived from $\Gamma^{(n)}$. The expression (10) is appropriate for numerical procedures involving matrix multiplications. 


\section{Displacement Coefficients: $P-S V$ Waves}

Let the source of plane waves be located in the half-space $z>z_{N}$ and let the potential of an incident $P$ wave be

$$
\phi_{i}^{(N+1)}=E(-1)^{N} \exp i\left(k x-\zeta_{N+1} \mathcal{E}-\omega t\right)
$$

The column matrix representing the source is

$$
\left(\begin{array}{c}
0 \\
0 \\
0 \\
\cdots \\
k \\
-\zeta_{N+1} \\
\left(\lambda_{N+1}+2 \mu_{N+1}\right) \zeta_{N+1}^{2}+\lambda_{N+1} k^{2} \\
-2 \mu_{N+1} k \zeta_{N+1}
\end{array}\right\} r E e^{-i \zeta_{N+1}{ }^{2} N}
$$

The factor $r$ is $\left(e^{-i \zeta_{n} z_{n}}, e^{-i \eta_{n} z_{n}}, e^{i \zeta_{n} z_{n}}, e^{i \eta_{n} z_{n}}\right)$ according as we solve for $\left(a_{n}, b_{n}\right.$, $\left.c_{n}, d_{n}\right)$. In the case of incident $S V$ waves the potential function for the inc dent wave is

$$
\psi_{i}^{(N+1)}=G(-1)^{N} \exp i\left(k x-\eta_{N+1} z-\omega t\right)
$$

then the column matrix representing the source is

$$
\left(\begin{array}{c}
0 \\
0 \\
0 \\
\cdots \\
\eta_{N+1} \\
k \\
-2 \mu_{N+1} k \eta_{N+1} \\
\mu_{N+1}\left(k^{2}-\zeta_{N+1}^{2}\right)
\end{array}\right) r G e^{-i \eta_{N+1} z_{N}}
$$

These column matrices are then inserted as replacements in the appropriate columns of (7). Then by the usual methods we obtain

$$
\begin{aligned}
& \Delta_{R} a_{n}=-\left(T_{W_{1} W_{2}}^{(0)} F_{\bar{W}_{1}}^{(1)} \bar{W}_{2} \bar{W}_{3} \bar{W}_{4} F_{W_{3} W_{4} W_{5} W_{6}}^{(2)} \cdots F_{W_{2 n-1} W_{2 n} 34}^{(n-1)} S^{(n)} S^{(n+1)} \cdots S^{(N-1)} S^{(N)} E^{\prime}\right. \\
& \cdot \exp \left(-i \zeta_{N+1} z_{N}-i \zeta_{n} z_{n}\right) \\
& =-\left(T^{\langle 0\rangle} F^{(1)} F^{(2)} \cdots F^{(n-1)}\right) S^{(n)} S^{(n+1)} \cdots S^{(N-1)} S^{(N)} E \\
& \cdot \exp \left(-i \zeta_{N+1} z_{N}-1 \zeta_{n} z_{n}\right)
\end{aligned}
$$


where, as before, the last indices in the summed parts of the numerator term $F^{(n-1)}$ may be $F_{\bar{w}_{2 n-1} \bar{W}_{2 n} 12}^{(n-1)}$ according as $n$ is even or odd. The multiplicative factors $S^{(p)}$ are the determinants of order four obtained from the last four columns of $\Lambda^{(p)}$ : $S^{(p)}=\left|\begin{array}{cccc}k & -\eta_{p+1} & k & \eta_{p+1} \\ \zeta_{p+1} & k & -\zeta_{p+1} & k \\ \left(\lambda_{p+1}+2 \mu_{p+1}\right) \zeta_{p+1}^{2}+\lambda_{p+1} k^{2} & 2 \mu_{p+1} k \eta_{p+1} & \left(\lambda_{p+1}+2 \mu_{p+1}\right) \zeta_{p+1}^{2}+\lambda_{p+1} k^{2} & -2 \mu_{p+1} k \eta_{p+1} \\ 2 \mu_{p+1} k \zeta_{p+1} & \mu_{p+1}\left(k^{2}-\zeta_{p+1}^{2}\right) & -2 \mu_{p+1} k \zeta_{p+1} & \mu_{p+1}\left(k^{2}-\zeta_{p+1}^{2}\right)\end{array}\right|$

ACKNowledgMents

This research was performed under NSF Grant G-10324.

\section{REFERENCES}

Dickson, L. E.,

1914. Elementary Theory of Equations, John Wiley and Sons, New York Haskell, N. A.,

1953. "The Dispersion of Surface Waves on Multilayered Media," Bull. Seism. Soc. Am., $43: 17-34$

Thomson, W.T.

1950. "Transmission of Elastic Waves Through a Stratified Solid Medium," J. Appl. Phys., $21: 89$.

Seismological Laboratory

California Institute of Technology

Pasadena, California

Contribution No. 1127

Manuscript received June 25, 1963. 